\title{
Pulmonary arterial hypertension or left heart disease with pulmonary hypertension? Toward noninvasive clarity, but time for a new paradigm
}

\author{
Robert P. Frantz \\ Affiliation: Mayo Pulmonary Hypertension Clinic, Rochester, MN, USA. \\ Correspondence: Robert P. Frantz, Mayo Pulmonary Hypertension Clinic, 200 First St SW, Rochester, MN \\ 55905, USA. E-mail: frantz.robertamayo.edu
}

\section{。} $@$ @RSpublications

PAH or left heart disease with PH? Perspective on group 1 and $2 \mathrm{PH}$ differentiators and a call for a unified approach http://ow.ly/OtRxU

Widespread availability of Doppler echocardiography, combined with increasing community awareness of pulmonary arterial hypertension (PAH) as an important and treatable disease, results in frequent referral of patients with suspected PAH to tertiary pulmonary hypertension centres. While this state of affairs is laudable, it does result in substantial numbers of patients who turn out not to have PAH being seen at tertiary pulmonary hypertension centres. In this issue of the European Respiratory Journal, JACOBS et al. [1] describe the retrospective development and prospective validation of a simple tool designed to improve accurate noninvasive distinction between PAH and pulmonary hypertension due to left heart disease (systolic, diastolic or valvular; World Classification of pulmonary hypertension group 2). This tool was derived from analysis of patients with PAH or group 2 pulmonary hypertension seen at a major European pulmonary hypertension centre. It may enhance noninvasive exclusion of group $1 \mathrm{PAH}$ in a subset of patients seen with pulmonary hypertension who have risk factors for group 2 pulmonary hypertension.

The tool utilises a combination of historical, echocardiographic and electrocardiographic parameters to create a risk score for presence of pulmonary hypertension related to left heart disease, defined as a pulmonary capillary wedge pressure or left ventricular end diastolic pressure $>15 \mathrm{mmHg}$, or rising above that level following administration of $500 \mathrm{~cm}^{3}$ i.v. saline. A risk score of $\geqslant 64$ had a positive predictive value of $92 \%$ for presence of pulmonary hypertension related to left heart disease with a specificity of $99 \%$, i.e. misclassifying one in $100 \mathrm{PAH}$ patients. A score of 64 can be achieved simply by having a clinical history of left heart disease combined with echocardiographic findings of left atrial enlargement and more than mild left valve disease.

A remarkably higher proportion of patients were confirmed to have PAH (79\% in the development cohort and $59 \%$ in the validation cohort) than would be the case in a community cohort, where group 2 pulmonary hypertension would be of much greater prevalence. Accordingly, the authors appropriately describe the need for validation of this tool in a community cohort prior to utilisation in clinical practice.

The authors settle on a risk score of 72 as a cut-off value beyond which there is no need to suspect PAH and to refer to a pulmonary hypertension centre, since one in 100 patients ultimately felt to have PAH had risk scores above 64 , and $100 \%$ specificity was felt important in order not to miss any PAH cases. This

Received: March 202015 | Accepted after revision: June 142015

The viewpoints expressed in this editorial are those of the author, and are not meant to represent the official viewpoint of the European Respiratory Journal or the National Heart Lung and Blood Institute.

Conflict of interest: Disclosures can be found alongside the online version of this article at erj.ersjournals.com

Copyright CERS 2015 
creates nice clean compartmentalisation: none of the true PAH patients would have been misclassified, and a subset of the group 2 patients would have been spared a pulmonary hypertension tertiary referral and a right heart catheterisation, if only the beleaguered community physician had access to this tool. While this achievement is satisfying, and fits with the desire to categorise utilising noninvasive tools, it does raise key questions. How does this tool compare with currently available tools? A series of models for differentiating PAH from HFpEF (pulmonary vascular disease associated with left-side heart failure and preserved ejection fraction) has previously been published, reinforcing the strong predictive value of historical characteristics supplemented by primarily simple echocardiographic parameters, including right atrial and left atrial size [2]. A purely echo-based tool that relatively effectively distinguishes pulmonary hypertension due to pulmonary vascular disease, a definition including groups 1,3 and 4, from pulmonary venous hypertension, is available [3]. This tool dichotomises patients based upon left atrial size, mitral E/e', and pulse wave Doppler of the right ventricular outflow tract (notching, acceleration time). Echocardiography can provide a wealth of morphological and haemodynamic information that should be exploited as fully as possible to help the clinician understand the most likely underlying haemodynamics [4]. The quality of community echocardiographic studies is tremendously variable, so it is important for the practitioner to demand high quality echocardiographic examinations that include the elements necessary to provide insight into haemodynamic profiles.

Perhaps most importantly, awareness of all of these analyses provides the clinician with a powerful understanding of the factors that influence the probability of a given patient falling into one category or another. This enhances noninvasive diagnostic accuracy, reducing the number of patients who require invasive haemodynamics, and lessening potential for inappropriate prescribing of PAH medications. The ability to distinguish clear group 2 pulmonary hypertension patients from possible group 1 remains a critical skill, given the nearly completely different current approaches to therapy, and the possibly differing tertiary areas to which referral may be most appropriate. In this regard, the authors may have given the clinician another useful tool, subject to validation in the community setting.

The present study does raise broader issues that deserve comment. What does a diagnosis of PAH mean in a patient with a risk score for group 2 pulmonary hypertension above 64, who must have a history of left heart disease, left atrial enlargement, and more than mild left valve disease by echocardiography? It does not require a risk scoring system for the average clinician to know that the overwhelming majority of patients with a history of left heart disease who have more than mild left valve disease and/or left atrial enlargement do not have PAH. 9\% of the patients classified by the authors as having PAH apparently had more than mild left valve disease, while $24 \%$ had left atrial dilation. Such patients, even if classified by experts as group $1 \mathrm{PAH}$, will generally behave differently with regard to response to and tolerability of PAH therapies. Indeed, patients with more than mild left heart disease are generally excluded from clinical trials of PAH therapies. There is also a trend toward ever more stringent haemodynamic inclusions for clinical trials, such as requirement for pulmonary capillary wedge pressure $\leqslant 12 \mathrm{mmHg}$ if the pulmonary vascular resistance is not that high and there are risk factors for left heart disease. Achieving a pure and clean PAH cohort is clearly desirable when doing clinical trials of novel PAH therapies, but leaves us increasingly bereft as we seek clarity regarding optimal application of these therapies in real world practice.

A remarkable $65 \%$ of the patients ostensibly referred out of concern for presence of PAH, who were ultimately classified as group 2 pulmonary hypertension, had more than mild left valve disease. This was based upon echocardiography done at the referral centre, leading one to wonder whether the valvular disease was known to be so extensive prior to referral. This may depend on the expertise of the community echocardiography laboratories. Was the referring provider really thinking that a patient with more than mild left valve disease had PAH? A clue may be found from the unusual haemodynamics in the patients confirmed to have group 2 pulmonary hypertension at right heart catheterisation. The transpulmonary gradient in the group 2 patients was $21 \pm 12 \mathrm{mmHg}$, with a pulmonary vascular resistance of 435 \pm 323 dyn $\cdot \mathrm{s} \cdot \mathrm{cm}^{-5}$. Right atrial pressure was $12 \pm 7 \mathrm{mmHg}$, tending to be higher than in the PAH patients.

Accordingly these patients were likely worrying their providers, perhaps being known to have left heart disease and/or valve disease, but clinically raising concern about mixed pre- and post-capillary pulmonary hypertension and right heart failure, well known risk factors for adverse outcome in left heart disease [5-7]. The community providers correctly recognised the high-risk nature of their patients. Referral of such patients to a tertiary centre seems very appropriate, albeit (and perhaps) such patients at some centres would be better served in the heart failure clinic than in the pulmonary hypertension clinic. However, as a cardiologist specialising in pulmonary hypertension but also caring for many patients with left heart failure or unexplained dyspnoea, and practising in a large tertiary multidisciplinary clinic, I often find both community providers and those at our institution turning to our pulmonary hypertension clinic staff for assistance in understanding and treating multifactorial pulmonary hypertension: the innumerable patients with mixed phenotypes. It is a source of pride to dig deep and find a way to help these patients. Step 1 is to 
carefully sort out all the possible contributors to their pulmonary hypertension; identifying sleep disordered breathing, finding the hidden cases of constrictive pericarditis, amyloidosis or occult pulmonary emboli that may have specific, useful therapeutic approaches. Incorrect diagnoses and unidentified causes of pulmonary hypertension are common. It is incumbent upon the expert pulmonary hypertension provider to fulfil the role of astute and compulsive diagnostician. Step 2 is to develop a customised treatment plan that addresses as many of the factors involved as possible: treating hypoxaemia and sleep disordered breathing, optimising left ventricular afterload reduction and diuretic management, identifying patients in need of left ventricular assist devices or transplantation. Trying to reduce the number of such patients seen in our pulmonary hypertension clinic is not one of our goals.

The definition of group $1 \mathrm{PAH}$ is not, and never will be, perfect. It is a clinical diagnosis supported by haemodynamic findings at a point in time. It is tremendously hampered by inaccessibility of the pulmonary vasculature to pathologic interrogation in the living patient. It neglects the frequently complex patients we face every day, with comorbidities that may impact the pulmonary arterial or venous vasculature, or which have implications for varying responsiveness to particular PAH therapies. It must be updated as clinical findings evolve, and as patients respond or deteriorate when treated with or without $\mathrm{PAH}$ therapy. Its weaknesses expose gaps in our understanding of pulmonary vascular and right ventricular pathophysiology across the spectrum of cardiopulmonary disorders. Fortunately the pulmonary hypertension community is finally starting to work on filling in the gaps.

\section{Heeding the call: the NHLBI Pulmonary Vascular Disease Phenomics Initiative}

Pulmonary hypertension is a risk factor for adverse outcome regardless of the World Classification in which a patient is felt to most closely fit. The current system, while serving us well, has also become a potential barrier to greater understanding. Progress has been made in understanding the prognostic implications of pulmonary hypertension across disease entities [1]. Advances in defining mixed pre- and post-capillary pulmonary hypertension, uncovering occult pulmonary vascular and left heart disease in the catheterisation laboratory $[8,9]$, and understanding lung pathologic changes in pulmonary hypertension associated with parenchymal lung disease have been substantial [10-12]. Sorely lacking are noninvasive markers of pulmonary vascular pathology, tools to detect ongoing vascular injury versus quiescence or healing, understanding of mediators of the right heart response to haemodynamic and environmental stressors, and myocardial targets for therapy. Also sorely lacking are agents that reverse the pulmonary vascular pathology, and that may be effective across varying forms of pulmonary hypertension.

Toward a unified model of pulmonary vascular disease and right heart response Step 1. Deconstruct the components of the pulmonary hypertension classification system.

Step 2. Utilise pulmonary vascular resistance and right ventricular function as unifying themes.

Step 3. Utilise holistic omics and network biology to detect homologies, differences.

Step 4. Identify novel pathways, biomarkers, and targets for therapy.

Step 5. Construct omic centric pulmonary hypertension classification system.

Step 6. Conduct trials centred on pulmonary vascular and right ventricular remodelling.

The National Heart, Lung, and Blood Institute Pulmonary Vascular Disease Phenomics (PVDOMICS) project (RFA-HL-027; clinicaltrials.gov) grew out of recognition of the prognostic impact of pulmonary hypertension across World Classification of pulmonary hypertension groups, combined with observation of the pulmonary vascular and right ventricular homologies and differences across groups. We seek to move from a therapeutic approach that solely utilises vasodilators with antiproliferative actions, which may cause adverse vasodilatory consequences in combined pre- and post-capillary pulmonary hypertension, to an approach based more upon anti-proliferative, pro-apoptotic, immunomodulatory and anti-inflammatory therapies, and also directly targeting the right ventricular response to pulmonary hypertension.

The PVDOMICS initiative will perform deep phenotyping across pulmonary hypertension groups, with meticulous assessment of clinical phenotype, haemodynamics, cardiac and pulmonary function. This will be combined with broad "omic" characterisation utilising the remarkable array of tools now available, including next generation sequencing, genome wide association studies, proteomic and metabolic analysis and micro-RNA characterisation [4, 13]. Network biology, bioinformatics and building of interactomes will identify new pathways and targets for therapy, and create a new way to classify the broad spectrum of patients we see in practice every day. This additional classification system will be complementary to, not in lieu of, the current highly useful system. It is hoped that this initiative will lead to clinical trials of therapies directed toward pulmonary vascular or right ventricular remodelling, targeting common pathways identified through the PVDOMICS project, and enrolling patients that cut across traditional 
World Classification groups, but who share similarities in right ventricular and pulmonary arterial pathophysiology and "omic" signatures.

\section{References}

1 Jacobs W, Konings TC, Heymans MW, et al. Noninvasive identification of left-sided heart failure in a population suspected of pulmonary arterial hypertension. Eur Respir J 2015; 46: 422-430.

2 Thenappan T, Shah SJ, Gomberg-Maitland M, et al. Clinical characteristics of pulmonary hypertension in patients with heart failure and preserved ejection fraction. Circ Heart Fail 2011; 4: 257-265.

3 Opotowsky AR, Ojeda J, Rogers F, et al. A simple echocardiographic prediction rule for hemodynamics in pulmonary hypertension. Circ Cardiovasc Imaging 2012; 5: 765-775.

4 Mazurek JA, Forfia PR. Enhancing the accuracy of echocardiography in the diagnosis of pulmonary arterial hypertension: looking at the heart to learn about the lungs. Curr Opin Pulm Med 2013; 19: 437-445.

5 Miller WL, Grill DE, Borlaug BA. Clinical features, hemodynamics, and outcomes of pulmonary hypertension due to chronic heart failure with reduced ejection fraction: pulmonary hypertension and heart failure. JACC Heart Fail 2013; 1: 290-299.

6 Lam CS, Roger VL, Rodeheffer RJ, et al. Pulmonary hypertension in heart failure with preserved ejection fraction: a community-based study. J Am Coll Cardiol 2009; 53: 1119-1126.

7 Kalogeropoulos AP, Georgiopoulou VV, Borlaug BA, et al. Left ventricular dysfunction with pulmonary hypertension: part 2: prognosis, noninvasive evaluation, treatment, and future research. Circ Heart Fail 2013; 6: 584-593.

8 Borlaug BA, Nishimura RA, Sorajja P, et al. Exercise hemodynamics enhance diagnosis of early heart failure with preserved ejection fraction. Circ Heart Fail 2010; 3: 588-595.

9 Tolle JJ, Waxman AB, Van Horn TL, et al. Exercise-induced pulmonary arterial hypertension. Circulation 2008; 118: 2183-2189.

10 Dournes G, Laurent F, Coste F, et al. Computed tomographic measurement of airway remodeling and emphysema in advanced chronic obstructive pulmonary disease. correlation with pulmonary hypertension. Am J Respir Crit Care Med 2014; 191: 63-70.

11 Peinado VI, Gómez FP, Barberà JA, et al. Pulmonary vascular abnormalities in chronic obstructive pulmonary disease undergoing lung transplant. J Heart Lung Transplant 2013; 32: 1262-1269.

12 Hoffmann J, Wilhelm J, Marsh LM, et al. Distinct differences in gene expression patterns in pulmonary arteries of patients with chronic obstructive pulmonary disease and idiopathic pulmonary fibrosis with pulmonary hypertension. Am J Respir Crit Care Med 2014; 190: 98-111.

13 Parikh VN, Jin RC, Rabello S, et al. MicroRNA-21 integrates pathogenic signaling to control pulmonary hypertension: results of a network bioinformatics approach. Circulation 2012; 125: 1520-1532. 